\title{
A Self-Organizing and Holonic Model for Optimization in Multi-Level Location Problems
}

\author{
Sana Moujahed, Nicolas Gaud, David Meignan \\ Multiagent System Group, \\ Systems and Transport Laboratory, \\ University of Technology of Belfort-Montbéliard, France
}

\begin{abstract}
Multi-Level Location Problems are generally considered as complex. To deal with these problems we propose an approach based on Holonic MultiAgent Systems (HMAS). HMAS have already proven to be a convenient way to engineer complex systems. This approach was merged with Artificial Potential Fields (APF) mechanims. The solution is obtained simultaneously with the holarchy. The holarchy is thus used to exploit and control the emergence of the solution. This solution is then evaluated to check its relevance according to global objectives represented thanks to a fitness function. This model was efficiently applied to a multi-level distribution system.
\end{abstract}

\section{INTRODUCTION}

T HE facility location ${ }^{1}$ problems have witnessed an explosive growth in the last four decades. As Krarup and Pruzan [1] point out, this is not surprising since location policy is one of the most profitable areas of applied systems analysis. This is due to the importance of location decisions which are often made at all levels of human organization. Then, such decisions are frequently strategic since they have consequential economic effects.

The term facility is used in its broadest sense. It refers to entities such as plants, bus-stops, schools, hospitals, fire stations, etc. The general problem is then the location of new facilities to optimize some objectives, such as distance, travel time or cost and demand satisfaction. In this paper we are interested in the the optimization of the distance from a demand to the nearest facility. This problem is referred to as the continuous p-median problem.

However, location problems are often extremely difficult to solve, especially to obtain an optimal solution (often classified as NP-Hard). Classical approaches are based on branch and bound, greedy heuristics, genetic algorithms, etc. These approaches are not easily adaptable to dynamic systems where system's constraints or data change. This is a real limitation since most of real problems are dynamic. To deal with this lack of flexibility and robustness, a multiagent approach is adopted. It is known to be well suited for dynamical problems [2], [3].

The choice of a multiagent approach provides several advantages. First, multiagent systems are well suited to model distributed problems. In such systems, several entities cooperate to fulfill collective and personnal goals. Second, even if the multiagent approach does not guarantee to find optimal solutions, it is often able to find satisfying ones without

${ }^{1}$ Location, positioning, deployment and siting are used as synonyms too much computational cost [4]. This paper attemps to demonstrate the relevance of the reactive multiagent approach for optimization in positioning problems. Then, it provides satisfying solutions in addition to other assets as flexibility, modularity and adaptability to open systems.

In this paper a holonic multiagent approach for multi-level facility location problem is proposed. It is based on the selforganization of reactive holons. From that perspective it is an original approach.

Multi-level location problems are particularly complex and classified as NP-Hard [5], since levels interact, influence each other, may have antagonist objectives and specific dynamics. The holonic paradigm and its application to multiagent systems are arising as one of the most adapted tools for the analysis and modeling of complex systems [6], [7].

The term holon was originally introduced in 1967 by the hungarian philosopher Arthur Koestler [8] to refer to natural or artificial structures that are neither wholes nor parts in an absolute sense. According to Koestler, a holon must respect three conditions: (1) being stable, (2) having the capability of autonomy and, (3) being able to cooperate. The stability means that a holon can react when strong perturbations occur. The autonomy implies that a holon is capable of self-management in order to achieve its own goals. The cooperation aptitude denotes that holons may work in common projects according to shared goals with other holons or other levels of holons. Holonic organizations have proven to be an effective solution to several problems associated with hierarchical self-organized structures [9], [10], [11], [12].

Our solution to deal with multi-level location problem, combines a holonic approach to artificial potential fields based mechanisms. Holon behavior is based on the combination of attractive and repulsive forces. The basic idea is that microscopic holon's behavior leads to the emergence of solutions at the macroscopic level [13]. Moreover, holonic model allows to control this emergence between the various levels and so facilitates the emergence of a consensus between objectives, that may be antagonist.

The structure of this paper reflects the above reported lines of thought. Hence, in sub-section II-A we introduce the general facility and the multi-level location problem. Subsection II-B details our generic organizational framework for holonic multiagent systems. Sub-section II-C presents the selforganizing approach for the location problem. Then, section III is devoted to the proposed self-organization framework for location problems and to its application to a multi-level 
distribution system. The last section gives some conclusions and perspectives.

\section{BACKGROUND}

\section{A. Location Problems Overview}

In literature, the general facility location problem is concerned with the determination of the optimal number, size and geographic configuration of facilities, in such a way that a certain criterion (or several criteria) is optimized.

Four nodal components characterize location problems [14]: (1) a space in which demands and facilities are located, (2) a metric that indicates distance (or other measures as time) between demands and facilities, (3) demands, which must be assigned to facilities, and (4) facilities that have to be located.

Three basic classes can be identified in location analysis: continuous location, network location and discrete location [15]. The differences between these arise from the structure of the set of possible locations for the facilities. Hence, finding optimal facility locations on the edges or vertices of a network corresponds to a network location model while in discrete location models the facilities can be placed only at a limited number of eligible positions. Finally, continuous location models are characterized by two essential attributes: (a) The solution space is continuous, that is, it is feasible to locate facilities on every point in the space. (b) Distance is measured with a suitable metric. Typically, the Euclidean distance metric or the Manhattan distance metric are employed.

Each of these three classes has been actively studied, arousing intense discussions on approaches proposed to solve location problems. This paper focuses on continuous problems and especially on multi-level location problems.

Many practical situations involve more than one type of facilities and therefore multi-level models have recently an increased deal of attention [15], [16]. Researchers give several names for this type of problems: hierarchical, multi-level, multi-echelon, multi-stage. Generally, the designation of the problem indicates the maximum number of levels considered: $\mathrm{k}$-hierarchical, k-level, k-echelon location problems refer to problems with, at most, $\mathrm{k}$ levels of facilities. The objective is to choose where to simultaneously locate facilities in each level in order to optimize a global objective. This problem is NP-hard, since it is a generalization of an NP-hard problem, as stated in [5].

A multi-level facility location model is needed whenever the facilities to be located can be grouped in levels. These levels may have different characteristics i.e. offering different services and interact with each other. So it is not possible to locate facilities in each level independently from the others.

Several examples exist in our daily lives that show the importance of considering multi-level facility location problems: the hierarchical health service system, the hierarchical education system, the multi-level structure of bank and postoffices, etc.

Various approaches were developed to tackle the different variants of the multi-level problem. These approaches are mostly dedicated to the static version of multi-level problem. [17] present a branch and bound algorithm for the multi-level uncapacitated facility location problem. Lagrangean relaxation is used in [18] to solve a two-hierarchical uncapacitated location-allocation problem. For the same problem [19] investigates dual-based procedures. In spite of the efficienty of these propositions to tackle multi-level problems, they are inexorably expensive in computation time, rarely useful for real-size problems. Mainly they lack robustness, scalability and adaptation to dynamic problems.

The next section is devoted to the description of a generic framework for Holonic Multi-Agent System, which will be applied in section III to control the emergence of a solution in multi-level location problem.

\section{B. A generic organizational framework for Holonic Multi- Agent System}

A holon is a self-similar structure composed of holons as sub-structures. This hierarchical structure composed of holons is called a holarchy. A holon can be seen, depending on the level of observation, either as an autonomous "atomic" entity or as an organization of holons. This duality is sometimes called the Janus Effect ${ }^{2}$, in reference to the two faces of a holon. A holon is a whole-part construct that is composed of other holons, but it is, at the same time, a component of a higher level holon. Examples of holarchies can be found in every-day life. Probably, the most widely used example is the human body. The body cannot be considered as a whole in an absolute sense. It is, in fact, composed of organs, that in turn are composed of cells, molecules, etc.

Holonic approaches have been applied to a wide range of applications. Thus, it is not surprising that a number of models and framework have been proposed for these systems [7], [20], [21]. However, most of them are strongly attached to their application domain and use specific agent architectures. In order to allow modular and reusable modelling that minimizes the impact on the underlying architecture, the framework is based on an organizational approach.

We have selected the framework presented in [3], [9], [22] based on the $\mathrm{RIO}^{3}$ meta-model [23] to represent organizations. We have leaned for this model since it enables formal specification, animations and proofs based on the OZS formalism [24].

In order to maintain the generic character of this framework [3] distinguishes between two aspects that overlap in a holon. The first is directly related to the holonic character of the entity, i.e. a holon (super-holon) is composed of other holons (sub-holons or members). This aspect is common to every holon, thus called holonic aspect. The second aspect is related to the problem that the members are trying to solve, and thus specific to the application or the domain of application.

A super-holon is an entity in its own right, but it is composed by its members. So, we need to consider how members organize and manage the super-holon. This constitutes the first aspect of the holonic framework. To describe this aspect, [3] defines a particular organization called Holonic Organization.

\footnotetext{
${ }^{2}$ Roman god with two faces. Janus was the god of gates and doorways, custodian of the universe and god of beginnings

${ }^{3}$ Role-Interaction-Organization
} 
This organization represents a moderated group in terms of roles and their interactions. To describe the status of a member inside a super-holon, it defines three main roles: Head, Part and Multi-Part. The Head role players are the representatives or moderators of the group, and a part of the visible interface. For the represented members we define two different roles. The Part role represents members belonging to only one superholon. The Multi-Part role is played by sub-holons shared by more than one super-holon.

In this approach, every super-holon must contain at least one instance of the Holonic Organization. Every sub-holon must play at least one role of this organization. This role defines its status in the composition of the super-holon.

Super-holons are created to satisfy objectives, and to perform certain tasks. To achieve these goals and tasks, the members must interact and coordinate their actions. The framework also offers means to model this second aspect of the super-holons. These goal-dependent interactions are modeled using organizations, called Internal Organizations, since they are specific to each holon and its goals/tasks. The behaviors and interactions of the members can thus be described independently of their holonic roles as a component of the super-holon. The set of internal organizations can be dynamically updated to describe additional behaviors. The only strictly required organization is the Holonic organization that describes member's status in the super-holon.

The framework is also concerned with a third important aspect of a Holonic MAS, the dynamics. Dynamics are inherent characteristics of MASs. The framework considers in particular two of the most attractive characteristics of Holonic MASs: how to regroup holons to create a superholons or join an exisiting super-holon, and self-organization. This requires holons to merge with other holons according to their ability to work together. To achieve that, the model is based on two important concepts: Affinity and Satisfaction. Affinity measures, according to the application's objectives, the aptitude of two holons to work together toward some shared objectives. It must be defined according to the domain of the application. Satisfaction measures the progress of the holon toward the achievement of its current goal. These two measures are intended to guide holons in selecting the most appropriate collaborators.

The framework guarantees a clear separation between the management of the super-holon and the goal-specific behaviors and favors modularity and re-usability. It also provides means to deal with dynamical aspect and allows to refine generic model according to the application domain. Section III will introduce a specialization of this framework to deal with optimization in multi-level location problem. The next subsection details the proposed self-organizing approach for the location problems.

\section{A Self-organized reactive approach for the continuous location problem}

Our model draws from the artificial potential fields approach which is a possible manner to build self-organized systems. This approach as well as the reactive one are presented in the next two sections, the proposed model is detailed in II-C.3.
1) The reactive approach: The reactive approach arose in computer science in the 80's with the work of M. Minsky [25]. Minsky constructs a thesis for a way in which human intelligence in all its complexity can be built up, layer by layer, from the interactions of simple parts called agents, who are themselves mindless. He describes the postulated interactions as constituting a "Society of Mind". This thesis matured in the end of 80's, when researchers where interested in the functioning of insects societies (termites, ants, bees). They showed that these reactive agents are able to collectively solve complex problems. This phenomenon observable at the macro level is called self-organization.

Self-organization exists in many natural systems and especially in insect societies. Such systems are composed of simple entities, for instance ants, which can solve complex problems without any global control [26]. Their organization results from the numerous interactions between agents and their environment. It is the environment that guides the agent behaviors and the whole system organization according to the so called stigmergy principle [13].

2) The artificial potential fields technique: Selforganization has been used to define decentralized algorithm to deal with path finding problems (ant algorithm [27]), collective tasks such as boxpushing [28], navigation [29], foraging with robots, etc. Most of these works are based either on digital pheromones, as inspired by ants, or on Artificial Potential Fields (APF). We adopt this second one because it is well suited to deal with spatial constraints as in the location problems.

The APF approach has several inspirations: physical, biological, etc. The concept was introduced by Kurt Lewin in his book "Principles of topological psychology" [30]. The basic idea is that human behavior is controlled by a force field generated by objects or situations with positive or negative values or valences.

During the past decade, potential field theory has gained popularity among researchers in the field of autonomous robots [31] and especially in robot motion planning thanks to their capability to act in continuous domains in real-time. By assigning repulsive force fields to obstacles and an attractive force field to the desired destination, a robot can follow a collision-free path via the computation of a motion vector from the superposed force fields [28]. In [32], artificial potential fields are used to tackle cooperation and conflict resolution between situated reactive agents.

3) A self-organizing multiagent model: In the proposed approach, facilities are modeled as reactive agents. These agents are situated in a finite and continuous environment. The behavior of an agent or a facility consists, first, in optimizing its position considering the perceived demands. Second, in considering interactions with other agents in order to reach the collective problem solving. These two features allow to deduce the holon satisfaction and affinity which are presented in sub-section III-A.

a) Local demand satisfaction: The agent's behavior consists in minimizing its distance from the perceived demand. The key idea is that a demand induces attraction forces which are applied on the agent. Considering one demand point, an 


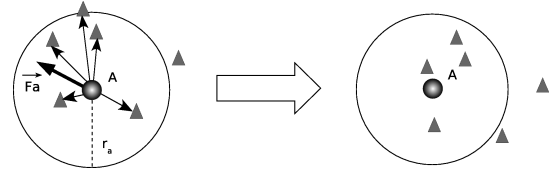

Fig. 1. Attractions lead the agent to the weighted barycenter of demands

attractive force is defined from the agent towards the demand. It is expressed as a vector the intensity of which is proportional to the demand weight and to the distance between the agent and the demand. Formally, for an agent $A$ perceiving a demand $D$ with weight $W_{D}$ :

$$
\vec{F}_{D / A}=W_{D} \cdot \overrightarrow{A D}
$$

The influence of the attraction decreases when the agent moves towards the demand. Thus, if the agent attains the demand the attraction behavior is inhibited.

For the set of perceived demands, the influence on an agent is defined as the sum of all induced forces. Formally, the local attraction force undergone by an agent $A$ is computed as follows:

$$
\vec{F}_{\text {demands } / A}=\frac{\sum_{i=1}^{n} \vec{F}_{i / A}}{n}
$$

Parameter $n$ is the number of demands perceived by the agent $A$ through its attraction radius $r_{a}(n=5$ in Fig. 1$)$. The demand is indexed by $i$.

Obviously, the radius $r_{a}$ should be considered as a variable. It can range from infinity to a calculated best value (specific to the problem). But in our real experiments we had a fixed radius imposed by the requirements. For this reason, in the following we will only deal with fixed radius.

The agent moves to the weighted barycenter of the demands, which is known to be the minimum average distance to several close weighted points [33], [34]. For example, if an agent is subject to two attractive forces (from two different demands), it will be more attracted towards the biggest demand. Then, it will move towards a balance point. This point is defined as the place where the two attraction forces are equilibrated.

When we have several agents, attraction forces may bring the agents to the same location. In such a case the process is sub-optimal since several agents cover the same demand. To prevent this process, repulsive forces are introduced to the model.

b) Local coordination: To prevent agents from having the same locations, repulsive forces between them are introduced. These forces concern close agents, i.e. situated in a given radius, defined as the repulsion radius ( $r_{r}$ in Fig.2).

The force intensity is defined as inversely proportional to the inter-agent distance.

Formally the repulsive force induced by an agent $B$ on an agent $A$ is expressed as follow:

$$
\vec{R}_{B / A}=\frac{\overrightarrow{B A}}{\|\overrightarrow{A B}\|^{2}}
$$

Then, the local repulsive force undergone by an agent $A$ is computed as follows:

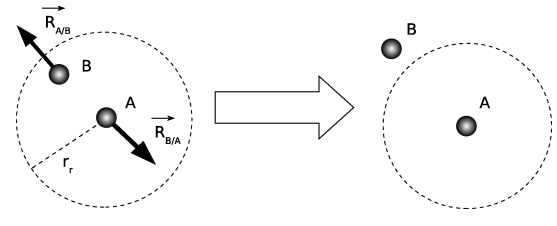

Fig. 2. Repulsions between agents $A$ and $B$ lead them to keep away

$$
\vec{R}_{\text {agents } / A}=\frac{\sum_{j=1}^{m} \vec{R}_{j / A}}{m}
$$

Parameter $m$ is the number of agents perceived by the agent $A$. These agents are indexed by $j$. Figure 2 illustrates this repulsive process between two agents.

Moreover, such repulsive forces allow to respect constraints on minimal distances separating facilities which are present in many facility location applications.

c) Collective solving: The agent behavior is defined as the weighted sum of both local attraction and repulsion forces. Formally, for an agent $A$, it is expressed as follows:

$$
\overrightarrow{\text { Move }}=\alpha \vec{F}_{\text {demands } / A}+(1-\alpha) \vec{R}_{\text {agents } / A}
$$

The coefficient $\alpha$ allows to favour either the attraction or the repulsion.

We now consider the whole system, where several facilities must optimize their positioning to cover numerous demands. In the self-organizing approach, no global control is used. Agents are created and randomly distributed in the environment and act following the defined individual behavior.

The collective solving process is presented in Algorithm 1.

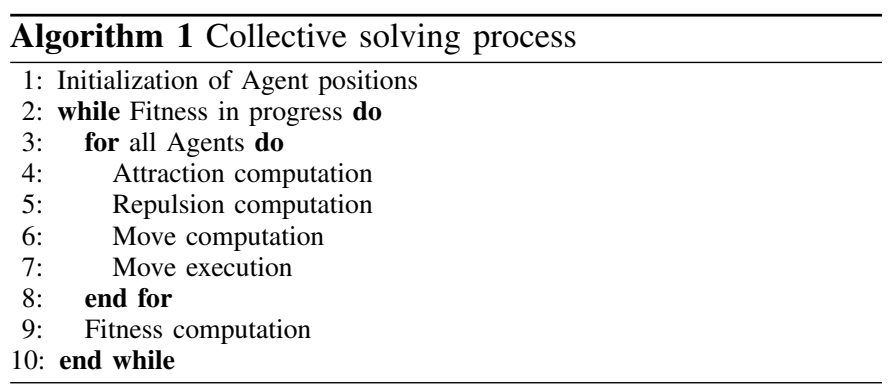

\section{ApPLICATION TO A MULTI-LEVEL FACILITY LOCATION PROBLEM}

\section{A. Holonic self-organization framework}

In this section, we detail how to define Affinity and Satisfaction (cf. sub-section II-B) to specialize the holons dynamics to solve the multi-level facility location problem. This dynamics stems from both attraction and repulsion forces.

We define two main kinds of holon's architecture: Demand Holon (the lowest level of the holarchy) and Facility Holons. Each holon will always search to join a super-holon to be supplied. Its basic behavior consists in integrating the nearest super-holon that it perceives. The affinity between a holon of level $n$ and a holon of level $n+1$ is thus defined according to the inverse of the distance separating them: the greater the 


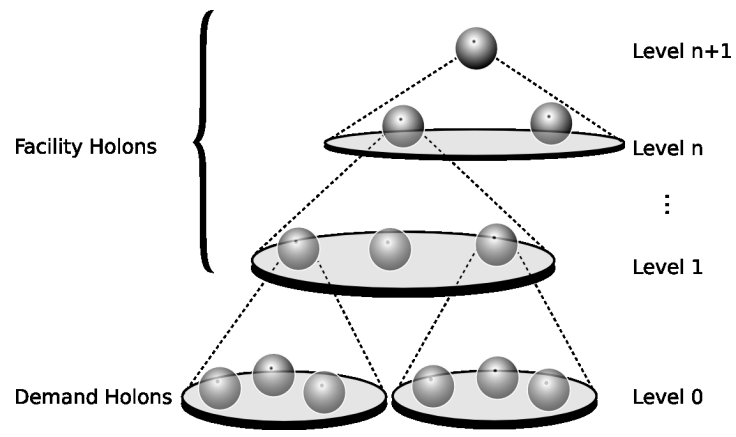

Fig. 3. The structure of the holarchy

distance, the lower the affinity. Otherwise, the affinity between two holons of the same level is defined according to the inverse of the distance to their super-holon.

In this problem the holarchy is mapped to a simple hierarchy where each level corresponds to a specific kind of holons. Each demand is assigned to a Demand Holon, these holons will then search to integrate a Facility Holon in charge of supplying them. Similarly, Facility Holons are then grouped into Facility holons (upper level), and so on. The whole process leads to the creation of the entire holarchy.

Figure 3 illustrates these various kinds of holons and a possible resulting holarchy.

The satisfaction of each holon must be defined. So, the selfsatisfaction $S_{i}$ (cf. equation 6) of a holon is defined according to the distance separating it from its super-holon.

$$
S_{i}=\frac{1}{d^{2}\left(X_{S H}, X_{i}\right)}
$$

With $X_{i}$ the position of the holon $i$ and $X_{S H}$ the position of its super-holon.

Thanks to Affinity, Satisfaction and Attraction-Repulsion forces (cf. sub-section II-C) we are able to define the behavior of each holon and thus create the holarchy. The solution is emerging from the structure of this final holarchy.

To evaluate this solution, the fitness of the entire holarchy must be computed. This global fitness $F$ (cf. equation 7) is obtained according to the fitness of each level in the holarchy, which is dependent on the fitness $F_{H_{(k, l)}}$ of each super-holon $H_{(k, l)}$ of the corresponding level 1 (cf. equation 8).

$$
F=\sum_{l=1}^{h} \sum_{k=1}^{n_{l}} F_{H_{(k, l)}}
$$

With $h$ the height of the holarchy and $n_{l}$ the number of holon in the level $l$.

$$
F_{H_{(k, l)}}=\frac{\sum_{m=1}^{n} M_{(m, l-1)} * d^{2}\left(X_{H_{(k, l)}}, X_{(m, l-1)}\right)}{\sum_{m=1}^{n} M_{(m, l-1)}}
$$

With $M_{(m, l-1)}$ the weight associated to the $m^{\text {th }}$ member of $H_{(k, l)}$ (level $\left.l-1\right), X_{H_{(k, l)}}$ the position of the $k^{t h}$ super-holon of level $l$ and $X_{(m, l-1)}$ the position of the $m^{\text {th }}$ member of $H_{(k, l)}$.

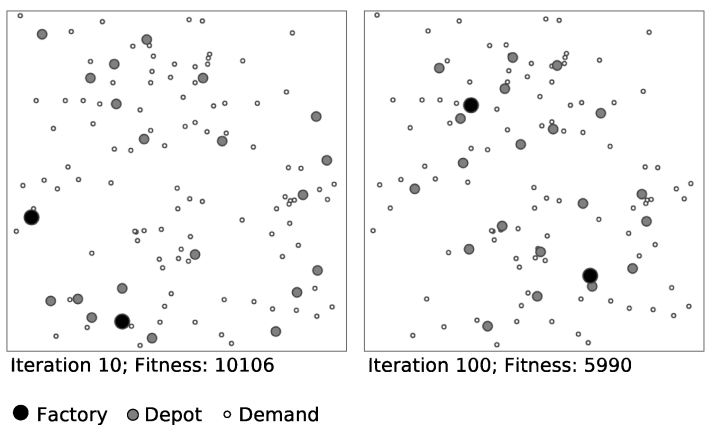

Fig. 4. Agents location for iterations 10 and 100

The solution of the multi-level location problem is obtained when each holon has reached a stable state, so that the sum of forces is null. This happens when the attraction forces from holon of the lower level $l-1$ and of the upper level $l+1$ and the repulsion forces from other holons of the same level $l$ are null.

Finally, thanks to these forces, we obtain a satisfying distribution of agents for each level of the holarchy.

\section{B. Experimental Results on a multi-level distribution system}

The potential field-based approach has already been applied to the classical version of location problem (1-level problem). A computational study has been presented in [35]. In this subsection we report the application of the previous model to a 2-levels distribution system.

In this system the lowest level corresponds to the Demands, level 1 coincides with the Depots and the highest one with the Factories.

Table I details the obtained results for different configurations (various number of demands, depots and factories). In this example, demands are randomly distributed over a 2D Euclidian space.

\begin{tabular}{|c|c|c|c|}
\hline $\begin{array}{c}\text { Demand } \\
\text { (level 0) }\end{array}$ & $\begin{array}{c}\text { Depot } \\
\text { (level 1) }\end{array}$ & $\begin{array}{c}\text { Factory } \\
\text { (level 2) }\end{array}$ & $\begin{array}{c}\text { Best found } \\
\text { solution }\end{array}$ \\
\hline 100 & 10 & 1 & 7477,72 \\
100 & 20 & 2 & 5869,9 \\
200 & 10 & 1 & 13839,45 \\
200 & 15 & 2 & 11284,58 \\
200 & 20 & 4 & 10513,04 \\
500 & 10 & 2 & 35860,98 \\
500 & 20 & 4 & 25468,75 \\
500 & 50 & 6 & 19600,98 \\
\hline
\end{tabular}

TABLE I

EXPERIMENTAL RESULTS OF 2-LEVELS DISTRIBUTION SYSTEM

Figure 4 illustrates the evolution of holons locations during the optimization process. It corresponds to the second configuration presented in the table I. Obviously, the holons repartition after 100 iterations is better than that presented after 10 iterations. This observation is confirmed by the fitness value.

The major objective of this paper consists in illustrating the relevance of the combination between the holonic approach 
and the APF-based holon architecture. The self-similar structure of the holarchy allows to dynamically add new levels without any significant modifications.

The main adavantage of this model is its self-adaptation aptitude and its capability to integrate problem's dynamics. Thus, the APF-based holon architecture enables to rapidely manage addition or suppression of entities (demand or facility) on any level without disturbing the system behavior.

\section{CONCLUSION}

In this paper a multi-level location problem is presented. It consists in simultaneously locate facilities in each level to fulfill a specified global objective. These levels interact, influence each other, and may have antagonist objectives. To deal with these problems a self-organization framework that combines a holonic approach to an APF-based mechanism is introduced.

Holon's behavior is defined as the conjunction of attractive/repulsive forces and satisfaction/affinity concepts. So, from an initial siting, the proposed approach allows holons to dynamically relocate themselves, in reaction to attractive and repulsive forces stemming from the demands and other holons and according to their satisfaction levels.

The underlying idea is that microscopic holon's behavior leads to the emergence of solutions at the macroscopic level. Moreover, holonic model allows to exploit this emergence between the various levels and so facilitates the emergence of a consensus between objectives.

This work confirms the growing potential of HMAS to tackle complex problems, and further works will be devoted to the adaptation of this model to other optimization domains.

\section{ACKNOWLEDGMENT}

The authors would like to thank Abderrafiâa Koukam, Stéphane Galland, Vincent Hilaire, Sebastian Rodriguez (Jefe) and Olivier Simonin for their help to achieve this work.

\section{REFERENCES}

[1] J. Krarup and P. M. Pruzan, "The simple plant location problem: Survey and synthesis," European Journal of Operations Research, vol. 12, pp. 36-81, 1983.

[2] J. Ferber, Multi-agent Systems : An Introduction to Distributed Artificial Intelligence. Addison Wesley, 1999.

[3] S. A. Rodriguez, "From analysis to design of holonic multi-agent systems : a framework, methodological guidelines and applications," $\mathrm{Ph} . \mathrm{D}$. dissertation, Université de Technologie de Belfort-Montbéliard, 2005.

[4] M. Wooldrige, Multiagent Systems - A Modern Approach to Distributed Artificial Intelligence. G. Weiss, 1999, ch. 1 Intelligent Agent, pp. 27-77.

[5] M. R. Garey and D. S. Johnson, Computers and Intractability, A Guide to the Theory of NP-Completeness. W.H Freeman and Co, 1979.

[6] F. Tecchia, C. Loscos, R. Conroy, and Y. Chrysanthou, "Agent behaviour simulator (abs): A platform for urban behaviour development," in GTEC'2001, 2001.

[7] M. Ulieru and A. Geras, "Emergent holarchies for e-health applications: a case in glaucoma diagnosis," in IECON 02 [Industrial Electronics Society, IEEE 2002 28th Annual Conference of the], vol. 4, no. 5-8, 2002, pp. 2957- 2961.

[8] A. Koestler, The Ghost in the Machine. Hutchinson, 1967.

[9] S. Rodriguez, V. Hilaire, and A. Koukam, "Towards a methodological framework for holonic multi-agent systems," in Fourth International Workshop of Engineering Societies in the Agents World, Imperial College London, UK (EU), October 2003, pp. 29-31.
[10] G. Bell, A. Cooper, M. Kennedy, and J. Warwick., "Using the holon framework : from enquiry to metrication - a higher education case study." in Proceedings of the 19th International System Dynamics Conference, Atlanta, Georgia., 2001.

[11] H.-J. Bürckert, K. Fischer, and G.Vierke, "Transportation scheduling with holonic mas - the teletruck approach," in Proceedings of the Third International Conference on Practical Applications of Intelligent Agents and Multiagents, 1998, pp. 577-590.

[12] G. Vierke and C. Russ, "Agent-based configuration of virtual entreprises," in Proceedings of the KI'98 workshop, 1998.

[13] H. V. D. Parunak and S. A. Brueckner, "Entropy and self-organization in multi-agent systems," in Fifth International Conference on Autonomous Agents, 2001, pp. 124-130.

[14] C. S. Revelle and H. A. Eiselt, "Location analysis: A synthesis and survey," European Journal of Operations Research, vol. 165, pp. 1-19, 2005.

[15] A. I. Barros, Discrete and Fractional Programming Techniques for Location Models. Kluwer Academic Pub, 1998.

[16] J. Dias, M. E. Captivo, and J. Climaco, "Dynamic multi-level capacitated and uncapacitated location problems: an approach using primal-dual heuristics," INESC-Coimbra, Tech. Rep., 2004.

[17] D. Tcha and B. Lee, "A branch-and-bound algorithm for the multilevel uncapacitated location problem," European Journal of Operations Research, vol. 18, pp. 35-43, 1984.

[18] S. Narula and U. Ogbu, "Lagrangean relaxation and decomposition in an uncapacitated 2-hierarchical location-allocation problem," Computers \& Operations Research, vol. 12, pp. 169-180, 1985.

[19] L. L. Gao and J. Robinson, "A dual-based optimization procedure for the two-echelon uncapacitated facility location problem," Naval Research Logistics, vol. 39, pp. 191-212, 1992.

[20] F. Maturana, W. Shen, and D. Norrie, "Metamorph: An adaptive agentbased architecture for intelligent manufacturing," International Journal of Production Research, vol. 37, no. 10, 1999, 2159-2174.

[21] J. Wyns, "Reference architecture for holonic manufacturing systems the key to support evolution and reconfiguration," Ph.D. dissertation, Katholieke Universiteit Leuven, 1999.

[22] S. Rodriguez, V. Hilaire, and A. Koukam, "Fomal specification of holonic multi-agent system framework," in Intelligent Agents in Computing Systems, International Conference on Computational Science (3), ser. LNCS, no. 3516, Atlanta, USA, 2005, pp. 719-726.

[23] V. Hilaire, A. Koukam, P. Gruer, and J.-P. Müller, "Formal specification and prototyping of multi-agent systems," in Engineering Societies in the Agents' World, ser. Lecture Notes in Artificial Intelligence, A. Omicini, R. Tolksdorf, and F. Zambonelli, Eds., no. 1972. Springer Verlag, 2000.

[24] P. Gruer, V. Hilaire, A. Koukam, and P. Rovarini, "Heterogeneous formal specification based on object-z and statecharts : semantics and verification." Journal of Systems and Software, vol. 70, no. 1-2, pp. 95105, 2004.

[25] M. Minsky, The Society of Mind. New York: Simon and Schuster, 1986.

[26] H. V. D. Parunak, "Go to the ant: Engineering principles from natural multi-agent systems," Annals of Operations Research, vol. 75, pp. 69$101,1997$.

[27] M. Dorigo and C. Blum, "Ant colony optimization theory: A survey," Theoretical Computer Science, vol. 344, no. 2-3, pp. 243-278, 2005.

[28] R. C. Arkin, Behavior-Based Robotics. MIT Press, 1998.

[29] C. W. Reynolds, "Flocks, herds, and schools: A distributed behavioral model," Computer Graphics, vol. 21, no. 4, pp. 25-34, July 1987.

[30] K. Lewin, Principles of topological psychology. McGraw-Hill Book Company, Inc, 1936.

[31] O. Khatib, "Real-time obstacle avoidance for manipulators and mobile robots," in IEEE international conference on robotics and automation, 1985, pp. 500-505.

[32] O. Simonin and J. Ferber, "Modeling self satisfaction and altruism to handle action selection and reactive cooperation," in Sixth International Conference on the Simulation of Adaptative Behavior, Paris, France, 2000, pp. 314-323.

[33] A. Likas, N. A. Vlassis, and J. J. Verbeek, "The global k-means clustering algorithm," Pattern Recognition, vol. 36, no. 2, pp. 451-461, 2003.

[34] F. Plastria, "On the choice of aggregation points for continuous p-median problems: a case for the gravity centre," TOP, vol. 9, pp. 217-242, 2001

[35] S. Moujahed, O. Simonin, A. Koukam, and K. Ghédira, "Self-organizing multiagent approach to optimization in positioning problems," in 17th European Conference on Artificial Intelligence, 2006, pp. 275-279. 